\title{
Spontaneous closure of vesicovaginal fistulas after bladder drainage alone: review of the evidence
}

\author{
Tony Bazi
}

Published online: 24 November 2006

(C) International Urogynecology Journal 2006

The abstract contained several orthographic errors that were introduced by the typesetter. The correct abstract is reproduced in full here.

\begin{abstract}
A vesicovaginal fistula may occur as a surgical complication, the result of obstructed labor, or a late manifestation of radiotherapy. Surgical treatment includes many routes and techniques, with a success rate reaching $100 \%$. The spontaneous closure of vesicovaginal fistulae following bladder drainage alone for varying periods has been reported by many. The factors favoring the success of this conservative method have not been well examined. In this investigation, all articles referring to this modality were
\end{abstract}

reviewed. The parameters studied included etiology, size, interval from causative insult to drainage, and duration of drainage. The incidence of spontaneous closure of fistulae after bladder drainage alone ranged between 0 and $100 \%$. Among all assessed criteria, the interval to drainage seems to have the best correlation with success. This finding is most likely explained on the basis of the epithelialization of the fistulous tract with time, preventing spontaneous healing. In view of the retrospective nature of the reviewed articles, the absence of a detailed classification system, and the heterogeneity of the treatment in question, no solid conclusion regarding management recommendations can be drawn.

The online version of the original article can be found at: http://dx.doi. org/10.1007/s00192-006-0194-7.

\section{T. Bazi $(\bowtie)$}

American University of Beirut,

8th Floor, 3 Dag Hammarskjold Plaza,

New York, NY 10017-2303, USA

e-mail: tb14@aub.edu.lb 